\title{
ÚLTIMAS PROPUESTAS SOBRE LA FINANCIACIÓN DE LOS PARTIDOS POLÍTICOS
}

\author{
PILAR CORTÉS \\ Universidad de Zaragoza
}

El vigente sistema de financiación de los partidos políticos españoles está configurado pór una serie de normas, como la Ley de partidos políticos y la LOREG, pero, principalmente, por la Ley $3 / 87$. En esta última se preve un sistema mixto de financiación pública y privada, el cual está integrado, por una parte, por tres tipos de subvenciones públicas, las electorales, las destinadas a cubrir los gastos de funcionamiento ordinario y aquellas que reciben los grupos parlamentarios de las distintas Asambleas representativas, y por otra, por las fuentes privadas que son las cuotas, donaciones, los rendimientos de las actividades propias del partido, los créditos que el partido concierte, los legados y las herencias que reciba y, en general, cualquier prestación en dinero o en especie que los partidos obtengan, todo ello corolado por un régimen de control que vela por su efectivo cumplimiento.

\section{PROPUESTAS DE REFORMA DE LOS DISTINTOS GRUPOS PARLAMENTARIOS}

Los escándalos que han ido salpicando la vida política española en los años noventa, han puesto de manifiesto la necesidad de revisar y reformar las normas que regulan la financiación de los partidos políticos ${ }^{1}$. Pues bien, si todas las fuer-

1. Una necesidad apuntada desde otros sectores de la sociedad, como es el caso del Círculo de Economía, que en un reciente documento, sugería la creación de una comisión de expertos indepen- 
zas políticas coinciden en esa necesidad, en lo que ya no están de acuerdo es en la orientación de esa reforma, es decir, en la dirección de esa modificación y en los puntos y criterios concretos que deberían modificarse.

En 1995, se creó en el Congreso de los Diputados, una Comisión de Estudio para la financiación de los partidos políticos, con el fin de que elaborase un texto consensuado para la esperada reforma. Tras la imposibilidad de conseguirlo, los distintos grupos parlamentarios fueron presentando, por separado, sus proposiciones de ley sobre financiación de partidos.

Desde junio hasta septiembre de 1996, se presentaron en el Congreso de los Diputados, un total de cinco proposiciones de Ley, firmadas respectivamente por el Grupo Parlamentario Socialista, el Popular, el Catalán, el Vasco y el Federal de Izquierda Unida-Iniciativa per Catalunya ${ }^{2}$. En ellas, como veremos a continuación, había puntos de convergencia, en los que los distintos textos coinciden, pero había también puntos de discrepancia en los que los textos se separaban, siendo éstos los que exigían un mayor debate.

Hay que destacar, en primer lugar, la justificación que cada uno de los grupos hace de su proposición, así como los objetivos a los que, según sus autores, responden.

Así, en la motivación de la proposición socialista, se pone el acento en la transparencia de la financiación y en el necesario control, señalando como triple objetivo, "conseguir que la financiación de los partidos sea: útil para los fines que la Constitución otorga a los mismos como expresión del pluralismo político y formación de la voluntad popular; transparente y pública a fin de que los ciudadanos puedan conocer, realmente, los ingresos y gastos de las formaciones políticas y quiénes y en qué cantidades contribuyen a su financiación y, por último, con control interno y externo a través del Tribunal de Cuentas para que se evite, lo máximo posible, cualquier veleidad de irregularidad en su financiación".

El grupo popular, por su parte, alude a la necesidad de garantizar y reformar los principios de suficiencia, transparencia y publicidad; recoger en un único texto legal el conjunto de normas que regulan el régimen tributario de los partidos políticos adaptándolo a sus características propias y establecer un marco fiscal más acorde con el papel que la Constitución Española atribuye a estas entidades.

El grupo catalán, pretende ampliar las vías de financiación privada y para ello aboga por "configurar un sistema en el que, sin abandonar la presencia de subvenciones estatales, se permita que las vías de financiación procedentes de las aportaciones de particulares individuales y de personas juridicas puedan facilitar el establecimiento de un sistema más eficaz y flexible y, a la vez, más. sensible al principio de publicidad y, por tanto, más próximo a la necesaria transparencia de las actuaciones económico-financieras de los partidos políticos".

dientes que estudie la reforma del actual sistema de financiación de las organizaciones políticas y emplaza a los partidos a incluir esa propuesta en sus programas electorales.

2. $B O C D, \mathrm{n}^{\circ} 39-1$, serie $\mathrm{B}$, de 14 de junio de $1996 ; B O C D$, n. ${ }^{\circ} 43-1,44-1$ y $45-1$, serie $\mathrm{B}$, de 4 de julio de $1996 ; B O C D$, n. ${ }^{\circ} 51-1$, serie $\mathrm{B}$, de 16 de septiembre de 1996. 
Para los vascos, su proyecto también muestra la preocupación por el control de la financiación, y para hacerle frente "trata de facilitar un modelo de financiación amplio y plural como es la Sociedad. Asimismo, trata de fomentar la transparencia limitando la financiación anónima y facilitando la posibilidad de que el Tribunal de Cuentas pueda actuar en las empresas que tengan relación con los partidos políticos con las técnicas de auditoría de aceptación general en todos los órdenes de la Sociedad".

$Y$, finalmente, el texto de Izquierda Unida-Iniciativa per Catalunya, sin marcar unos objetivos concretos, insiste en la necesidad de configurar un sistema de financiación de los partidos, como un factor esencial para garantizar la eficacia del sistema democrático, y que establezca las garantías y medios precisos para que ese sistema no incorpore elementos de distorsión entre la voluntad popular y el ejercicio del poder político, distorsión que se produciría si los partidos tuvieran que responder, no sólo de las exigencias de sus afiliados y electores, sino también de las de sus eventuales patrocinadores, motivo por el cual rechazan la liberalización total en materia de financiación.

En general, todas las formaciones coinciden en la prioridad de dotar de transparencia, publicidad y del adecuado control al sistema de financiación de los partidos, sistema que debe ser suficiente para que los partidos cumplan las funciones que constitucionalmente tienen asignadas.

$\mathrm{Si}$ estos son los objetivos que las proposiciones apuntan, veamos ahora el contenido del articulado con las medidas concretas para conseguirlos.

En una primera aproximación, todas las proposiciones mantienen el sistema mixto de financiación, pública y privada.

En cuanto a la financiación pública, lo primero que hay que destacar es el mantenimiento de sus fuentes de financiación casi en los mismos términos que en la actual Ley, como enseguida veremos.

Respecto a las subvenciones estatales para gastos de funcionamiento ordinario, todos coinciden en la necesidad de obtener representación parlamentaria para tener acceso a dichas subvenciones, siendo los resultados de las elecciones al Congreso de los Diputados, la referencia para esa distribución. De nuevo, y tal y como ocurre en la actual ley, la representación obtenida en el Senado no es tenida en cuenta a la hora de calcular la subvención correspondiente.

Sin embargo, a la hora de repartirlas, con la actual legislación se divide la cantidad total en tres partes, una de ellas se asigna según los escaños, y las otras dos se asignan según los votos, pero sólo se computan los obtenidos en aquellas circunscripciones en las que se hubiera obtenido el $3 \%$ de los votos válidos. Pues bien, las propuestas de socialistas, populares, catalanes y vascos, mantienen estos criterios, pero es Izquierda Unida la que propone modificarlos y plantea su sustitución por el criterio único del total de los votos obtenidos por cada partido en las últimas elecciones al Congreso de los Diputados.

El dinero público destinado a hacer frente a dichas subvenciones que, como no podía ser de otro modo, irá recogido en una partida presupuestaria incluida en los Presupuestos Generales que cada año apruebe el Parlamento, debería ser actualizado anualmente según un criterio objetivo. Pues bien, a este respecto sólo 
los Grupos Socialista e Izquierda Unida incluyen en sus propuestas esa previsión, señalando el Índice de Precios al Consumo como el referente para llevar a cabo la citada actualización. El resto de los grupos no menciona esta medida pero, no obstante, como apunta Pilar del Castillo, se trata de una cuestión tan obvia que no hay razón para creer que suponga un obstáculo para el diálogo y las negociaciones de una futura ley³.

De igual manera, coinciden socialistas e Izquierda Unida, en incluir dentro de las fuentes de financiación pública, las subvenciones extraordinarias para realizar las campañas de propaganda que puedan establecerse en la Ley Orgánica reguladora de las distintas modalidades de Referéndum.

Salvadas estas diferencias entre las diversas proposiciones, hay una serie de novedades en las que coinciden todos los textos, de las cuales nos hacemos eco seguidamente.

Es lugar común de todos los grupos, aunque con pequeñas diferencias de matiz, considerar subvenciones públicas algo que ya lo era de hecho, y que el Tribunal de Cuentas incluso ya había llamado la atención sobre la cuestión. Se trata de las subvenciones públicas que reciben los Grupos de Cargos Electos de Entidades Locales, según se establezca en las normas reguladoras de esos órganos representativos. Y además, de esos fondos, los textos establecen la posibilidad de que dichos Grupos hagan aportaciones a sus partidos.

En general, también prevén que los Grupos Parlamentarios, tanto de las Cortes Generales como de las Asambleas Legislativas de las Comunidades Autónomas, realicen aportaciones a sus partidos, procedentes de las subvenciones que reciben. Se trata, en definitiva, de recoger legalmente una circunstancia que viene siendo practicada por los grupos y los partidos, desde el inicio de la etapa democrática.

Es el grupo vasco, el que introduce una última novedad, cual es, la posibilidad de que los partidos reciban subvenciones públicas para sus gastos de funcionamiento ordinario pero, además del consignado en los Presupuestos Generales del Estado, también procedente de los presupuestos de las Comunidades Autónomas. La distribución de esas subvenciones sería, igualmente, con arreglo a los votos y escaños obtenidos por el partido en las últimas elecciones, pero en este caso, a la Asamblea Legislativa de que se trate. No obstante, la proposición vasca es la única que prevé esta medida, de lo que se podría deducir que no iba a ser fácil que dicha previsión se incluyera en una futura ley.

Pues bien, de lo visto hasta ahora, podemos comprobar que, en torno a la financiación pública, los distintos textos presentados no suponen una radical reforma, más bien al contrario, se percibe una cierta línea de continuidad, en la cual, por cierto, parecen estar prácticamente de acuerdo todas las fuerzas políticas ${ }^{4}$.

3. Del Castillo Vera, P.; "La financiación de los partidos políticos: 1977-1997", RCG n. $41,1997$.

4. En este punto, observamos cómo ninguno de los textos presentado por los distinto Grupos, se hace eco del debate abierto en foros internacionales, con respecto a la igualdad de mujeres y hombres en cuanto a sus derechos políticos, y más en concreto en las condiciones de acceso a los cargos públicos, en cuya defensa se están proponiendo medidas que podrían afectar al sistema de financiación actual de los partidos políticos. Se trata de la posibilidad de adoptar medidas que premien, eco- 
Sin embargo, ese elogiable acuerdo respecto a la financiación pública, se torna discrepancia cuando se trata de diseñar el modelo de financiación privada.

En principio, todos coinciden en la necesidad de contemplar, junto a la financiación pública, una financiación privada, y de ahí que en todos los textos estén previstas una serie de contribuciones con ese origen. Además, se intuye el interés de las distintas fuerzas políticas en potenciar la financiación privada a la vista de las bonificaciones fiscales que prevén para las cuotas de los afiliados y para las donaciones. Ventajas fiscales que pretenden persuadir al ciudadano, para que contribuya al sostenimiento económico del partido político con el que se sienta más identificado.

Dentro de esas medidas se incluye la deducción fiscal de la cuota de afiliado y de las donaciones, del Impuesto sobre la Renta de las Personas Físicas.

En líneas generales, se mantienen las actuales fuentes de financiación privada: cuotas de afiliados, donaciones, rendimientos de patrimonio, herencias o legados y créditos o préstamos. Pero, a pesar de esa inicial apariencia de acuerdo, surgen discrepancias en torno a las contribuciones procedentes de personas jurídicas y en torno al carácter anónimo o nominal que deben tener, en general, los fondos de origen privado.

La posibilidad de recibir aportaciones privadas de personas jurídicas está prevista en las proposiciones de los grupos popular, catalán y vasco, cada uno de ellos con distintos matices.

La proposición del grupo popular contempla, simultáneamente, las aportaciones de personas físicas y jurídicas, sometiéndolas a los mismos límites. Así, establece que la suma total de las aportaciones que haga una misma persona, física o jurídica, en un mismo año natural a un partido político, no podrá ser superior a la cantidad de 15.000.000 de pesetas ni podrá superar el 15\% del presupuesto anual de dicho partido. La única referencia específica a las aportaciones de personas jurídicas, es la exigencia de acuerdo adoptado en debida forma por el órgano social competente al efecto.

nómicamente, la incorporación de mujeres en las listas, o de penalizar, con disminución de la subvención pública correspondiente, a aquellas fuerzas políticas que no incluyan mujeres en sus listas. En este sentido, la Convención sobre la eliminación de todas las formas de discriminación contra la mujer, de 18 de diciembre de 1979 ( $B O E$ de 21 de marzo 1984), no considera discriminación, el hecho de que los Estado adopten medidas especiales de carácter temporal encaminadas a acelerar la igualdad "de facto" entre el hombre y la mujer. Y, más concretamente, en la Conferencia de Nueva Delhi, celebrada entre el 14 y el 18 de febrero de 1997, se examinaron una serie de medidas de acción positiva dirigidas a reforzar la presencia de las mujeres en los parlamentos, y entre ellas, que los partidos presenten, sistemáticamente, un tercio de candidaturas femeninas y les conceda un tercio de sus fondos electorales; que los partidos políticos y las instituciones financieras internacionales dispongan de fondos especiales para contribuir a las campañas electorales de candidaturas femeninas; que en los países donde se prevén ayudas o subvenciones con fondos públicos a los partidos políticos se tomen medidas de fomento, por ejemplo, haciendo que la cuantía percibida sea proporcional al número de mujeres candidatas y/o electas y que, finalmente, en los países en los que se conceden subvenciones a los grupos parlamentarios, pueda concedérseles una prima en función de la proporción de mujeres que contenga el grupo. Se ha utilizado el texto mecanografiado de la Ponencia que Teresa Freixes Sanjuán expuso en el Congreso de la Asociación Española de Derecho Constitucional, celebrado en Alicante (marzo 1999), bajo el título: La igualdad entre las mujeres y los hombres, la no discriminación y las medidas de acción positiva en el derecho internacional y comunitario". 
La proposición del grupo vasco, admite la posibilidad de que las personas jurídicas contribuyan a la financiación de los partidos, sin incluir en su texto tratamiento específico para ellas, ya que las equipara a las de las personas físicas, para las que no prescribe un límite máximo.

El grupo catalán, incluye la posibilidad de que los partidos reciban fondos tanto de personas físicas como jurídicas, con el límite de que la cantidad aportada por una misma persona no sea superior al 15\% del presupuesto anual del partido político beneficiado.

En contra se sitúan los grupos socialista y el de IU. La proposición socialista simplemente no hace referencia a las aportaciones de personas jurídicas, ni las nombra, mientras que IU las prohíbe expresamente.

En cuanto a las aportaciones anónimas, también varía la postura de los distintos grupos.

La proposición popular, permite aportaciones anónimas tanto de personas físicas como jurídicas, con el límite de que las que reciba un partido no podrán superar en un ejercicio económico anual el $10 \%$ de la cantidad asignada en los Presupuestos Generales del Estado en ese ejercicio para la subvención pública de los partidos políticos.

En iguales términos se expresa la proposición vasca, permitiendo las contribuciones anónimas y recogiendo la misma limitación máxima que el texto popular.

El texto catalán, no permite explícitamente las aportaciones anónimas, pero no exige en ningún momento la identificación de los donantes.

De nuevo, son los textos de los grupos socialista e IU los que se colocan en contra de los tres anteriores. La proposición socialista exige, en todo caso y para cualquier cuantía, la identificación de los donantes, sin dejar lugar, por tanto, para las donaciones anónimas.

Y el texto de IU, recoge la expresa prohibición de que el partido reciba aportaciones anónimas.

Son pues, los dos puntos sobre los que descansa la falta de acuerdo entre las distintas formaciones políticas, razón por la cual el texto aprobado por la Ponencia de la Comisión no ha conseguido, como veremos más adelante, la aprobación unánime de los representantes de todos los Grupos Parlamentarios ${ }^{5}$.

No queremos acabar este acercamiento a las distintas proposiciones sin hacer una referencia al régimen de control y fiscalización que los distintos textos incorporan.

Los cinco textos prevén un control interno y un control externo. El interno lo llevaría a cabo el propio partido, y en cuanto al externo, si bien presente en todas las proposiciones, surge alguna discrepancia en cuanto al tribunal competente.

Mientras la proposición socialista y la de IU atribuyen en exclusiva la competencia al Tribunal de Cuentas, las otras tres proposiciones, popular, vasca y catalàna, incluyen una fórmula en la cual, si bien conceden la com-

5. Se trata del Texto elaborado por la Ponencia de la Comisión Constitucional, hecho público el día 5 de marzo de 1998, al que más adelante haremos referencia. 
petencia al Tribunal de Cuentas, lo hacen sin perjuicio de las competencias atribuidas a los órganos de fiscalización de las Comunidades Autónomas previstos en sus respectivos Estatutos, de manera que se produciría una dispersión en una tarea en la cual, a nuestro parecer, resulta fundamental conseguir la máxima unificación.

La elaboración de un informe por parte del Tribunal, en el que se ponga de manifiesto la regularidad y adecuación a la Ley de las cuentas de los partidos, es una exigencia que recogen los cinco textos.

Además de eso, la propuesta catalana todavía contempla la posibilidad de que los partidos sometan sus cuentas a una auditoría externa con carácter previo a la fiscalización del Tribunal de Cuentas o del órgano autonómico equivalente.

En el ámbito del control, preven los textos de populares, socialistas e IU, la obligación del Gobierno de elaborar un Plan Especial de Contabilidad para partidos políticos que recoja la especificidad de éstos y en el que se identifiquen los diferentes centros de imputación de ingresos y gastos en que puedan estructurarse los partidos a nivel territorial. Si bien el texto vasco no incluye ninguna medida en este sentido, el catalán autoriza al Gobierno a dictar cuantas disposiciones de aplicación y desarrollo de la ley sean necesarias, de manera que se podía entender que no plantearía oposición a la elaboración de ese Plan Especial.

También en cuanto al régimen sancionador, tantas veces denunciado a lo largo de nuestro trabajo, aparecen algunas discrepancias importantes.

Las propuestas popular y vasca, dejan las cosas como están en la actualidad, no ponen en manos del Tribunal ni una sola medida sancionadora que pueda disuadir a los partidos de incumplir la Ley. Hablan de exigir responsabilidades, pero no especifican quién responderá, ni en qué consistirá esa exigencia.

La proposición catalana, va un poco más allá y prevé que el Tribunal pueda imponer sanciones pecuniarias, de acuerdo con lo que reglamentariamente se establezca. De manera que habrá que esperar a que se regule reglamentariamente la cuestión para saber de qué sanciones se está hablando.

Son las proposiciones socialista y de IU las que contienen, en idénticos términos, un régimen sancionador, especificando dos medidas sancionatorias concretas:

- En el caso de que un partido obtenga donaciones que contravengan las limitaciones y requisitos que marque la Ley, la sanción aplicable consistirá en una multa en cuantía equivalente al doble de la aportación ilegalmente percibida.

- Para el caso de que un partido, salvo causa justificada, no presente las cuentas correspondientes al último ejercicio anual o éstas sean tan deficientes que impidan al Tribunal de Cuentas llevar a cabo su cometido fiscalizador, el partido infractor no recibirá la subvención anual para sus gastos de funcionamiento.

Se trata, a nuestro juicio, de unas medidas ajustadas y proporcionadas si pretendemos que los partidos cumplan con las previsiones que la Ley exige.

Y todavía incluyen, socialistas e IU, una última medida sancionatoria, dentro de las Disposiciones transitorias. Se trata de la posibilidad de suspender la percepción de la subvención anual para gastos de funcionamiento ordinario, si no 
acreditan haber cumplido con la obligación de los partidos, prevista en la misma Disposición transitoria, de adaptar sus estatutos y normas internas a lo dispuesto en la futura nueva Ley; en el plazo de un año.

No obstante, si esta previsión ya supondría un avance en el régimen sancionador de las organizaciones partidistas, en ninguno de los textos encontramos la propuesta de instar al Gobierno para que reforme el Código Penal en el sentido de crear, expresamente, un tipo delictivo de financiación ilegal de los partidos políticos de manera que, además de especifiar el procedimiento y el órgano sancionador correspondientes, el Tribunal pueda dar traslado al Ministerio Fiscal, si tuviera indicios de su infracción ${ }^{6}$.

Finalmente, socialistas e IU pretenden extender la aplicación de la Ley a aquellas asociaciones sin ánimo de lucro que creen los partidos políticos o sus afiliados, para el desarrollo de objetivos de interés público vinculados a sus actividades. Medida que aparece reflejada en las proposiciones catalana y popular, si bien sólo remite a la Ley de financiación para la regulación de las donaciones de personas físicas y jurídicas.

Pues bien, este es el sistema de financiación que cada una de las propuestas considera adecuado. Seguidamente, sería la Comisión la que estudiase y trabajáse sobre ellas, con el fin de proponer un único texto al Congreso.

\section{PROPOSICIÓN DEL TEXTO UNIFICADOR}

Las cinco proposiciones fueron enviadas a la Comisión Constitucional para su estudio y para intentar llegar a un acuerdo sobre un texto único. En la Comisión, y siguiendo el procedimiento habitual, se nombró una Ponencia que se encargaría de informar todas y cada una de las proposiciones?

Tras estudiar las iniciativas, la Ponencia elaboró un informe, en el cual proponía a la Comisión un texto aprobado por una mayoría integrada por el Grupo Popular, Grupo Catalán, Grupo Vasco y Grupo de Coalición Canaria ${ }^{8}$.

6. En este sentido se pronunció Carlos Jiménez Villarejo (fiscal jefe de las Fiscalía Especial para la Represión de los Delitos Económicos Relacionados con la Corrupción), considerando que las infracciones en materia de financiación de partidos son "conductas de contenido lesivo del orden constitucional que deben tipificarse penalmente ; El País, 17 de diciembre de 1998. Esa misma línea ha mantenido la Fiscalía Anticorrupción, cuyo Fiscal Jefe solicitó, en el Informe presentado en el mes de mayo de 1999, la tipificación expresa del delito de financiación ilegal de los partidos políticos, pues en la vigente regulación, concretamente en lo tocante al régimen sancionador, "resulta imposible precisar cuál es el procedimiento y cuál el órgano sancionador". Se ha solicitado, por escrito, el citado a la Fiscalía Anticorrupción, pero tras obtener el silencio por respuesta, se ha utilizado la información publicada en el diario 'El País, 8 de mayo de 1999.

7. La Ponencia estaba formada por los diputados Del Burgo Tajadura, Trías Sagnier y La Puerta Quintero, por el Grupo Parlamentario Popular; Gimeno Marín y Fernández Marugán, por el Grupo Parlamentario Socialista; Santiso del Valle, por el Grupo Parlamentario de Izquierda Unida-Iniciativa per Catalunya; López de Lerma y López, por el Grupo Parlamentario Catalán; Mardones Sevilla, por el de Coalición Canaria; González de Txabarri, por el Grupo Parlamentario Vasco y Lasagabáster Olazábal por el Grupo Parlamentario Mixto.

8. BOCD n. ${ }^{\circ} 139-1$, serie $\mathrm{B}$, de 5 de enero de 1998. 
El texto de la Ponencia, señala como objetivos básicos de la nueva regulación, unificar en un único texto el conjunto de normas que regulan el régimen tributario de los partidos adaptándolo a sus características propias, y establecer un marco fiscal más acorde con el papel que nuestra Constitución les atribuye. Objetivos que, por otra parte, coinciden con los que señalara la proposición del grupo popular.

En cuanto a la regulación concreta y respecto a la financiación pública, mantiene el régimen que proponen, en líneas generales, todas las proposiciones. Contempla las subvenciones electorales, las subvenciones para gastos de funcionamiento ordinario, las subvenciones de los Grupos Parlamentarios, incluyendo los del Parlamento Europeo, e incorpora, como novedades, las subvenciones que reciben los grupos de representantes en los órganos de las Administraciones Locales, las subvenciones para gastos de funcionamiento ordinario que puedan conceder las Comunidades Autónomas o las Entidades Locales y las subvenciones extraordinarias para realizar campañas de propaganda. Además, mantiene el doble criterio de distribución de votos y escaños.

Respecto a la financiación privada, admite las aportaciones de personas jurídicas, concediéndoles el mismo tratamiento que a las de personas físicas, y fijando el límite máximo que cualquiera de ellas puede aportar a un partido en un mismo año natural en la cantidad de 25.000.000 de pesetas (en la actualidad son 10.000.000).

En ningún precepto se permiten ni se prohiben expresamente las aportaciones anónimas. No obstante, cuando el artículo 7 habla de la justificación de las cuotas y aportaciones, exige que se abonen en cuentas de entidades de crédito abiertas exclusivamente para dicho fin, las que son obligatorias de los afiliados, así como de las aportaciones de militantes y simpatizantes, en cuantías que no excedan de 50.000 pesetas. Pero, además, sigue el artículo, las restantes aportaciones privadas deberán abonarse en una cuenta distinta a la anterior y deberá dejarse constancia del nombre completo del donante, identificación que no se exigía en las otras aportaciones.

Pues bien, nosotros entendemos que es en este punto donde aparece la posibilidad de que algunas aportaciones sean anónimas porque, si bien las cuotas y aportaciones obligatorias de militantes no necesitan de la exigencia de identificación, las aportaciones de militantes y simpatizantes cuyo importe no supere las 50.000 pesetas son voluntarias y no van acompañadas de la exigencia de la identificación del donante, y de ahí se deduce que las donaciones anónimas estarían permitidas hasta una cuantía máxima de 50.000 pesetas. Cifra, por otra parte, no muy elevada, a la que podrían acceder un buen número de ciudadanos si quisieran contribuir al mantenimiento económico de un partido y desearan permanecer en el anonimato, sin olvidar que se trata de aportaciones físicas, nunca jurídicas.

$\mathrm{Y}$, finalmente, respécto al tercer punto que nos interesa, la fiscalización y el control, mantiene el doble control, interno y externo, atribuyendo este último en exclusiva al Tribunal de Cuentas, pero sin perjuicio de las competencias atribuidas a los órganos de fiscalización de las Comunidades Autónomas previstas en sus propios Estatutos. Se trata de una concesión lógica si tenemos en cuenta que el texto ha sido apoyado por los representantes elegidos en las listas de partidos autonómicos. 
Hasta ahora, la obligación de rendir cuentas al Tribunal, era únicamente para los partidos con representación en el Congreso de los Diputados, mientras que en el nuevo texto, esa obligación se extendería a los partidos que tengan representación en cualquiera de las instituciones públicas. De manera que, en principio, se puede ver incrementado el número de fuerzas políticas que presentarán sus cuentas al Tribunal.

El plazo fijado, finalmente, para que los partidos presenten sus cuentas al Tribunal es de seis meses a partir del cierre de cada ejercicio, extendiéndose las cuentas anuales consolidadas a los ámbitos estatal, autonómico y provincial?

No obstante lo dicho, para la rendición de cuentas de los Grupos Parlamentarios de las Cortes Generales y de las Asambleas Legislativas de las Comunidades Autónomas, se remite a lo que dispongan sus respectivos Reglamentos. Reglamentos que la única previsión que tienen en este sentido es la obligación de llevar una contabilidad específica de la subvención que recibe el Grupo Parlamentario, y la obligación de ponerla a disposición de la Mesa de la Cámara correspondiente, pero tan solo en el caso de que ésta lo pidiera.

Importante novedad que incorpora el texto incluído en el informe de la Ponencia, es el régimen sancionador que introduce, concediendo al Tribunal de Cuentas la competencia de imponer determinadas sanciones a los partidos políticos que incurran en una violación de la propia Ley.

De ese modo, si un partido obtiene donaciones que contravengan las limitaciones y requisitos que la Ley establece, el Tribunal podrá acordar la imposición de una multa de cuantía equivalente al doble de la aportación ilegalmente percibida, que será deducida del siguiente libramiento de la subvención anual para sus gastos de funcionamiento. Y si, por otra parte, un partido no presenta las cuentas correspondientes al último ejercicio anual, o éstas son tan deficientes que impiden al Tribunal llevar a cabo su cometido fiscalizador, éste podrá acordar que no le sean libradas al infractor, las subvenciones anuales para sus gastos de funcionamiento.

Dejando ya de lado el articulado del texto propuesto por la Ponencia, en su parte dispositiva, también introduce una serie de medidas que no podemos pasar por alto.

Es el caso de la Disposición adicional séptima, donde se prevé la adecuación anual, como mínimo, al incremento del Índice de Precios al Consumo, del importe global de la consignación que se incluya en los Presupuestos Generales del Estado para atender a las subvenciones que la ley regula en su artículo 3 (subvenciones anuales no condicionadas para atender los gastos de funcionamiento ordinario). Era una previsión incluida en las proposiciones socialista y de IU, sobre la que adelantábamos nuestra confianza en que no sería objeto de controversia.

Prevé, además, que el Gobierno elabore y remita a las Cortes Generales, previo informe del Tribunal de Cuentas, un proyecto de Ley de reforma del régimen contable y del control y fiscalización de la actividad financiera de los partidos

9. El plazo variaba en las distintas proposiciones: nueve meses en la socialista, seis meses para catalanes, vascos, populares e IU. 
(Disposición Final segunda), y que apruebe, también previo informe del Tribunal de Cuentas, un Plan Especial de Contabilidad para los partidos políticos.

En realidad, se trata de un texto unificador de las cinco proposiciones que, a grandes rasgos, lo que ha hecho ha sido articularlo incorporando las distintas novedades que aquellas apuntaban. A pesar de eso, el texto no ha sido aprobado por la totalidad de los miembros de la Ponencia ${ }^{10}$.

Aunque el texto fue aprobado por una mayoría en la Ponencia, y pese a que la proposición podría haber salido adelante con los votos de los grupos que la apoyaban, no sería conveniente que una Ley como esta fuese aprobada por tan solo una parte de las fuerzas políticas ${ }^{11}$.

Se trata de una Ley buscada durante mucho tiempo, cuya necesidad es reconocida por todos los partidos políticos, por eso, pretender aprobarla en contra de alguno de ellos no parecía lo más adecuado. Nuestros representantes deberían continuar trabajando y negociando, para intentar conseguir un texto totalmente consensuado, sin olvidar que, por otro lado, de la redacción final de la normativa que, en su caso llegase a aprobarse, depende en gran medida "la revitalización democrática de nuestro sistema político y parte importante de la credibilidad social de la forma de gobierno" 12 .

Sería deseable que el talante de acuerdo y de consenso que presidió la presentación y aprobación de la actual Ley de financiación, estuviese presente también en la nueva redacción, y que ahora, en la nueva legislatura, se reinicien los trabajos de acercamiento para conseguirlo.

Lo que es imprescindible es voluntad política para seguir adelante, y si esa voluntad se presupone, es de esperar que el acercamiento de las distintas posturas será, tal vez lento, pero posible ${ }^{13}$.

\section{ALGUNOS PROBLEMAS PLANTEADOS CON LA NUEVA PROPOSICIÓN}

El nuevo texto propuesto por la Ponencia, mantenía como criterios de reparto de las subvenciones públicas para gastos de funcionamiento ordinario, el número de votos y de escaños obtenidos por el partido. E igualmente, mantenía que

10. La razón última del desacuerdo está en lo relativo a las aportaciones anónimas y las aportaciones de personas jurídicas, ambas fuentes de financiación privada.

11. Esa incapacidad de las fuerzas políticas para sacar adelante una nueva Ley de financiación, ha sido calificada por Ramón VARGAS-MACHUCA ORTEGA, como "uno de los fracasos más sonados de esta legislatura. El País, 6 de febrero del 2000.

12. Presno Linera, M. A., "La reforma del sistema de financiación de los partidos políticos", $R D C$, n. ${ }^{\circ} 57,1999$.

13. En los programas electorales del PP, PSOE y CIU, con ocasión de su concurrencia a las elecciones generales del 12 de marzo del 2000, está presente la necesidad de modificar el sistema de financiación de partidos creando una nueva ley, cuya base ha de ser el rigor y la transparencia. Además, proponen modificar las normas electorales para disminuir los gastos de campaña y abogan por reforzar y ampliar las competencias del Tribunal de Cuentas en el procedimiento de control y sanción de las cuentas de los partidos. 
para su asignación, la correspondiente consignación presupuestaria se dividirá en tres cantidades: una de ellas se distribuirá en proporción al número de escaños obtenidos por cada partido político en las últimas elecciones al Congreso de los Diputados, y las otras dos restantes proporcionalmente a todos los votos obtenidos en dichas elecciones, siempre y cuando hayan sido obtenidos en distritos electorales en los que haya superado la cota del $3 \%$ de los votos válidos.

De manera que, al igual que ocurre con la actual legislación, se vuelve a plantear el problema de la discriminación entre partidos parlamentarios y extraparlamentarios, en primera instancia, y entre partidos parlamentarios mayoritarios y minoritarios, en segunda.

De nuevo, se discriminan los votos de aquellos partidos que han superado la barrera del $3 \%$ de los votos válidos en un distrito electoral. Y, después, en la fase de reparto de escaños, se vuelve a premiar a los que más votos obtuvieron porque el reparto de escaños va en función de aquellos.

Se trata de unos criterios de distribución denunciados por la doctrina y por algún partido, pero a los que no dudan en acudir las distintas fuerzas políticas en sus proposiciones, con la única excepción de IU.

Los partidos mayoritarios se ven favorecidos, o al menos no salen perjudicados, con esos criterios. Y los partidos nacionalistas también se benefician en el sentido de que, como ellos concurren a las elecciones en unos distritos electorales concretos, obtienen todos sus votos en esos distritos, superando ampliamente la barrera del $3 \%$, de manera que rentabilizan sus votos, por utilizar términos económicos, tanto en la asignación de la subvención por votos como por escaños.

Sin embargo, las formaciones políticas más pequeñas que presentan sus listas en la totalidad de distritos electorales, tienen tan dispersados sus votos, que se encuentran con problemas a la hora de conseguir superar el $3 \%$ de votos válidos en cada distrito, para poder tener acceso a las subvenciones públicas. Y si no consiguen los votos necesarios, tampoco podrán después obtener escaños, con lo cual otra vez se quedarán sin la subvención correspondiente.

Se trata de un problema planteado ya en la tramitación de la actual regulación y al cual, el texto propuesto por la Ponencia, sigue sin dar solución.

En segundo lugar, sigue sin hacerse referencia al Senado a la hora de repartir la subvenciones públicas. El nuevo texto, en idénticos términos que la legislación vigente, tiene en cuenta solamente los votos y escaños obtenidos al Congreso de los Diputados.

No obstante, siendo una discriminación denunciada por la doctrina, todas las fuerzas políticas coinciden en dejar fuera de los criterios de reparto la representación del Senado, como se pone de manifiesto en las distintas proposiciones, no haciendo mención a la Cámara Baja.

Finalmente, nada se dice en la propuesta sobre los órganos del partido que recibirán la subvenciones públicas. No se refleja si serán los órganos centrales o si, por el contrario, serán los centros regionales o locales los que recibirán las ayudas económicas que les correspondan. Podemos deducir que, ante el silencio del texto, seguirán siendo los órganos centrales los que reciban todo el dinero procedente de las subvenciones y luego, desde ahí, se distribuyan entre los demás centros. 